\title{
FAST POWER LINE DETECTION AND LOCALIZATION USING STEERABLE FILTER FOR ACTIVE UAV GUIDANCE
}

\author{
Yuee $\operatorname{Liu}^{\mathrm{a}}{ }^{*}$, Luis Mejias ${ }^{\mathrm{a}}$, Zhengrong $\mathrm{Li}^{\mathrm{b}}$ \\ ${ }^{a}$ Cooperative Research Centre for Spatial Information and Australian Research Centre for Aerospace Automation at \\ Queensland University of Technology, 22-24 Boronia Road, Eagle Farm, Brisbane, QLD, 4009 Australia - \\ (yuee.liu,luis.mejias)@qut.edu.au \\ ${ }^{\mathrm{b}}$ ROAMES, Ergon Energy, 61 Mary Street, Brisbane, QLD 4000, Australia - eric.lizr@ roames.com.au
}

Commission III, Working Group VII

KEY WORDS: line detection, steerable filter, oriented filter, Gaussian kernel, power line, UAV guidance

\begin{abstract}
:
In this paper we present a fast power line detection and localisation algorithm as well as propose a high-level guidance architecture for active vision-based Unmanned Aerial Vehicle (UAV) guidance. The detection stage is based on steerable filters for edge ridge detection, followed by a line fitting algorithm to refine candidate power lines in images. The guidance architecture assumes an UAV with an onboard Gimbal camera. We first control the position of the Gimbal such that the power line is in the field of view of the camera. Then its pose is used to generate the appropriate control commands such that the aircraft moves and flies above the lines. We present initial experimental results for the detection stage which shows that the proposed algorithm outperforms two state-of-the-art line detection algorithms for power line detection from aerial imagery.
\end{abstract}

\section{INTRODUCTION}

In recent years, there has been a considerable interest in civilian applications of unmanned aerial vehicles (UAVs), especially in electrical infrastructure inspection and corridor monitoring applications (Li, et al., 2010;Mills, et al., 2010; Rathinam, et al., 2008). UAVs for this type of tasks offer benefits such as reduction in the cost per kilometre of infrastructure inspected. However, currently they have payload limitations in size, weight and power (SWaP). For this reason, in some circumstances onboard sensors used typically for inspection or data collection can be seamlessly used for navigation purposes. This duality of purpose provides benefits when it comes to performing a more accurate inspection task. Most existing UAV guidance approaches assume that the location of network assets is known and that GPS can provide highly accurate real-time position information. However in practice, network GIS data available is often incomplete, inaccurate or even non-existent in some regions. Vision-based method provides a good alternative for navigating UAVs directly over power line networks, especially in GPS-denied environments. In vision-based active UAV guidance, images taken from a onboard video camera are used to estimate the relative position of the plane with regards to the objects and assist the UAV navigation. The challenge for vision-based navigation is the real-time detection and localization aspects.

Objects of interest used for UAV guidance vary from case to case. In this paper, we consider object of interest power lines, and therefore their location in the sensor frame is proposed for UAV guidance. Using power lines to achieve active UAV guidance will be very beneficial to perform more advanced and optimal aerial inspection tasks. From the sensor perspective, a power line is a linear feature with specific width and length that changes with the sensor height.
Linear feature detection is an important field in computer vision and has been intensively investigated in the past few years (Akinlar \& Topal, 2011;Nieto, et al., 2011; Von Gioi, et al., 2010). Most of the recently proposed methods are based on either gradient/edge (Akinlar \& Topal, 2011;Fernandes \& Oliveira, 2008;Nieto, et al., 2011;Von Gioi, et al., 2010) or ridge/valley information(Jang \& Hong, 2002;Koller, et al., 1995;Steger, 1998). Another well known approach is the Hough transform (Hough, 1962). More recently, a fast version called kernel-based Hough transform has been proposed by using an efficient voting scheme, but produces infinitely long lines rather than line segments (Fernandes \& Oliveira, 2008). Moreover, it detects more false positive lines and the line position is shifted from the original position. At gradient/edge level, Line Segment Detector (LSD) is a technique that uses connected component analysis (CCA) on quantized gradient orientation to obtain colinear pixels, then calculate eigenvector of these pixels as line segment (Von Gioi, et al., 2010). Although LSD produces accurate line segment, the involved region growing on the whole image makes it computationally expensive and unsuitable for real-time applications. Another gradient/edge approach is the Edge Drawing algorithm (EDLines) which extracts lines from the edge pixel chains based on the least squares line fitting method (Akinlar \& Topal, 2011). It is the fastest line segment approaches to the best of our knowledge. However, both of them respond to all kinds of edges and generate two edge lines for a line, which make them unsuitable for power line detection. Some authors have considered lines as an object with two parallel edges. Koller et al. used two firstorder Gaussian derivative for the left and right line sides respectively, and combined the response of the two filters in a nonlinear way as the final response of line (Koller, et al., 1995). Line can also be estimated by extracting the centre line or ridge. Steger computed ridge points by approximating the image with

\footnotetext{
* Corresponding author.
} 
a quadratic polynomial of image convolutions with Gaussian derivatives(Steger, 1998). Steger's method can detect centre line with subpixel precision. Similarly, Jang and Hong extracted ridge points from the distance map generated by performing Euclidean distance transform on the edge map, but distance transform is slow for a large image (Jang \& Hong, 2002).

Generally speaking, existing line detection approaches either suffer from heavy computational cost or fail to obtain robust feature detection performance. Some so-called "real-time" line detection methods often generate too many false positives while the object semantics have seldom been considered. In this paper we have evaluated several state-of-the-art line detection algorithms for power line detection from aerial imagery. A new real-time power line detection algorithm is proposed by using steerable filters as well as prior knowledge of objects surrounding the environment where the power line is.

\section{THE STEERABLE FILTER}

2D oriented filters, such as Gaussian filter and Gabor filter, have been widely used for robust edge and ridge detection (Casasent \& Smokelin, 1994;Liu \& Dai, 2006;Mehrotra, et al., 1992). One approach is to convolve the oriented filter with the image at each orientation and analyse each filter response. Rotating filters at many directions is time consuming, especially if one filter is different from another by some small rotations. To address this computational cost, Steger proposed steerable filters (Steger, 1998). In this section, the idea of steerable filter is briefly introduced.

Steerable filters are based on a small number of basis filters defined at pre-specified orientations, the filter response rotated at arbitrary direction is synthesized from the linear combination of these basis filters. These filters can be rotated efficiently by the proper interpolation of basis filters. Given $f(x, y)$ as the filter response, and $f^{\theta}(x, y)$ as the filter response rotated at the angle $\theta$, the steerable filters can be written in Equation 1.

$$
f^{\theta}(x, y)=\sum_{i=1}^{M} k_{i}(\theta) f^{\theta_{i}}(x, y)
$$

where $\quad \theta_{i}=$ the $i^{\text {th }}$ basis angle, $i \in 1,2, \ldots, M$

$k_{i}(\theta)=$ the $i^{\text {th }}$ interpolation function

$f^{\theta_{i}}(x, y)=$ the $i^{\text {th }}$ basis function

$M=$ the number of basis filters

Gaussian derivatives are widely used in computer vision due to their desirable properties such as steerability. Additionally they are band-pass filters which reinforces the response along its direction while suppress the response orthogonal to its orientation. Generally odd-order filters are used for edge detection, while even-order filters are for ridge detection, the second-derivative Gaussian is chosen as we focus on ridge detection for power line detection. The filters are formed by steerable quatrature pair filters - a second-derivative Gaussian and its Hilbert transform. This is able to determine the line type and direction.

Let $G_{2}$ be the steerable filter, its quadrature pair steerable quadrature filter $\mathrm{H}_{2}$ is the approximation to the Hilbert transform of $G_{2} . H_{2}$ is achieved by finding the least squares fit to a polynomial times a Gaussian in $(x, y)$. It is found that the satisfactory approximation could be achieved by using a third order, odd parity polynomial. It means that four basis filters suffice to steer the quadrature filter $\mathrm{H}_{2}$ at any arbitrary orientation, and $G_{2}$ requires three basis functions. Figure 1 illustrates the basis filters of $G_{2}$ and $H_{2}$. The steerable quadrature filter pairs at any direction $\theta$ are implemented:

$$
G_{2}^{\theta}=\sum_{i=1}^{3} k_{i}^{G}(\theta) G_{2}^{\theta_{i}} \quad H_{2}^{\theta}=\sum_{i=1}^{4} k_{i}^{H}(\theta) H_{2}^{\theta_{i}}
$$

where $G_{2}^{\theta}, H_{2}^{\theta}=G_{2}$ and $H_{2}$ rotated by an angle $\theta$

$k_{i}^{G}(\theta)=$ the $i^{t h}$ interpolating function for the steerable filter, they are $\cos ^{2}(\theta),-2 \cos (\theta) \sin (\theta), \sin ^{2}(\theta)$

$G_{2}^{\theta_{i}}=$ the $i^{\text {th }}$ basis filters for the steerable filter.

$k_{i}^{H}(\theta)=$ the $i^{t h}$ interpolating function for quadrature filter, $\cos ^{3}(\theta),-3 \cos ^{2}(\theta) \sin (\theta), 3 \cos (\theta) \sin ^{2}(\theta), \sin ^{3}(\theta)$

$H_{2}^{\theta_{i}}=$ the $i^{\text {th }}$ basis filters for the quadrature filter

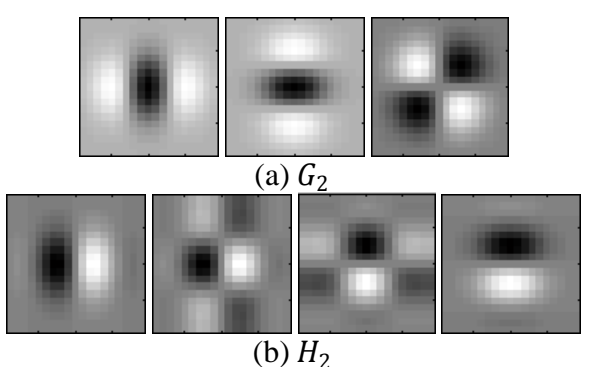

Figure 1. The basis filters of $\boldsymbol{G}_{\mathbf{2}}$ and $\boldsymbol{H}_{\mathbf{2}}$

In the implementation of steerable quadrature pair filters, the first step is to create 2D basis filters. Gaussian function $G(x, y)$ is the unique rotationally symmetric function with the linearly separable property, i.e. $G(x, y)=g(x) * g(y)$. To facilitate the computation, the first and second derivatives of Gaussian function and their Hilbert transform is calculated in one dimension as well as the polynomial fitting of Hilbert transformation.

2D filter convolution with the image is implemented by convolving each row in the image with the horizontal projection, and then convolving each column with the vertical projection.

\section{POWERLINE DETECTION ALGORITHM}

In this section, the proposed power line detection algorithm is presented. The main idea is to obtain the ridge points rather than edges based on the energy functions of the steerable oriented filter, and then extract linear features by grouping the collinear ridge points. In addition, knowledge is used to distinguish power lines from surrounding linear objects. The characteristics are summarized as follows: 1) symmetry: geometry similarity along the main axis. The left and right sides have similar magnitude. 2) elongation: averagelength $\gg$ average width. 3) parallelism: the left and right borders should be locally parallel. 4) homogeneity: the region should be homogeneous, and the profile of the region intensities resembles a Mexican hat.

\subsection{Ridge Points Identification}

Power line segments can be identified by detecting the ridge points of the linear patterns. Most previous power line detection methods used edge detection-based method, which we believe are not appropriate because: 1) a thick power line segment represented with more than two pixels in an image will be 
detected as two parallel edge lines after edge detection; 2) due to the complexity of the environment (e.g. nearby linear objects such as fences and roads) many false positive edge lines will also be detected. In this paper, we propose oriented Gaussian filter to detect ridge points in image.

It is well known that Gaussian derivatives perform well to detect elongated structures with small width variations along their axes, due to 1) they are band-pass filters which suppress lines whose width is smaller than a threshold; 2) all pixels within the filter kernel are used, which have advantages in suppressing the noise (Cai, 2011;Koller, et al., 1995;Steger, 1998). In this section, two energy functions, oriented energy and ridge energy, are analyzed and compared for determining the ridge points.

The two-dimensional Gaussian function, $G(x, y)$ is utilized:

$$
G(x, y)=e^{-\left(\frac{x^{2}+y^{2}}{2 \sigma^{2}}\right)}
$$

where $\sigma=$ the standard deviation which depends on the line's width. It is recommended to set $\sigma$ as $w / \sqrt{3}$ in which $w$ is the line width (Steger, 1998). The kernel size of Gaussian is set to $\pm 3 \sigma$ without losing discernible accuracy.

Let $G_{2}^{\theta}$ and $H_{2}^{\theta}$ be the steerable quadrature pair filters at the angle $\theta, \otimes$ be the convolution operation, the convolution of the pair filters with the image $I$ can be obtained as:

$$
G_{2}(\theta)=G_{2}^{\theta} \otimes I \quad H_{2}(\theta)=H_{2}^{\theta} \otimes I
$$

The oriented energy $E_{2}(\theta)$, which is the combination of response of $G_{2}^{\theta}$ and $H_{2}^{\theta}$, is defined as (Steger, 1998):

$$
E_{2 o}(\theta)=\left[G_{2}(\theta)\right]^{2}+\left[H_{2}(\theta)\right]^{2}
$$

For any pixel $j$, there exist a largest oriented energy at an angle $\theta_{d}$. This angle is the principal orientation of the pixel $j$.

$$
\theta_{d o}=\underset{\theta}{\arg \max } E_{2 o}(\theta)
$$

In order to extract ridge points and exclude more edge points, the ridge energy function $E_{2 r}(\theta)$ is defined as (Cai, 2011):

$$
E_{2 r}(\theta)=\left\{\begin{array}{cc}
0 & {\left[G_{2}(\theta)\right]^{2}-\left[H_{2}(\theta)\right]^{2}<0} \\
{\left[G_{2}(\theta)\right]^{2}-\left[H_{2}(\theta)\right]^{2}} & \text { otherswise }
\end{array}\right.
$$

The profile of the steerable quadrature filter $H_{2}(\theta)$ is for edge detection, while the shape of steerable filter $G_{2}(\theta)$ is for ridge detection. Ridge points would increase the response of $G_{2}(\theta)$ and suppress the response $H_{2}(\theta)$. The ridge energy could be treated as the ridge strength of a pixel. If pixel $j$ 's ridge energy is smaller than 0 , then pixel $j$ is categorized to edge pixels, otherwise it is ridge pixel. The orientation, at which pixel $j$ has the largest ridge energy, is the principal orientation of the pixel.

$$
\theta_{d r}=\underset{\theta}{\arg \max } E_{2 r}(\theta)
$$

The steerable quadrature pair filters reinforce the energy along the direction of the pair filters while suppress the energy perpendicular to the direction, thus the ridge points would have larger energy than their surrounding pixels. The procedure of identifying the ridge points 1 ) calculating the oriented and ridge energy $E_{2}(\theta, j)$ for each pixel $j$ at each orientation by steering the quadrature pair filters, and then 2) setting the largest value of oriented energy and ridge energy $E_{2}\left(\theta_{d}, j\right)$ functions as the value of the pixel $j$. If $E_{2}\left(\theta_{d}, j\right)$ is larger than a pre-defined threshold, pixel $j$ is classified as the ridge point. In this paper, we divide $[0, \pi]$ into 8 directions $\theta=(i / 8) \pi, i=0,1, \ldots, 7$ for computational efficiency, and locate the largest energy value from these 8 directions.

An example of ridge point detection is given in Figure 2 in which column (a) represents the original image, and column (b) and column (c) are the ridge point detection results by oriented filter and ridge energy respectively. As convolution of the quadrature filter pairs involves all pixels in the kernel, it is clear to see that the noise is filtered out as well as the thin lines whose width is smaller than the width of the interest line. It should be noticed that the ridge detector only run once and the ridge is not thinned out to be one pixel width as other method do. The reason is that: 1) a power line might be too weak to be thinned, otherwise power line could be broken into to many fine segments which makes further long, difficult and inaccurate detection process. 2) performing the ridge detector to detect one pixel width ridge significantly increases the computational burden, which is not suitable for the real-time application.

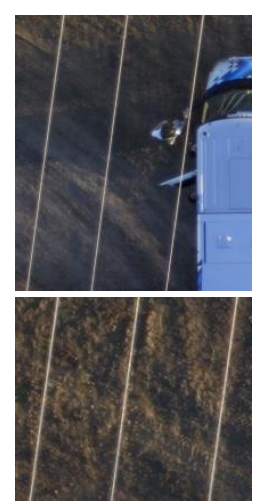

(a)

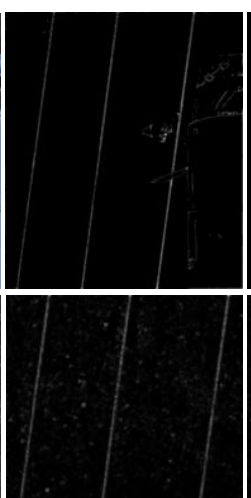

(b)

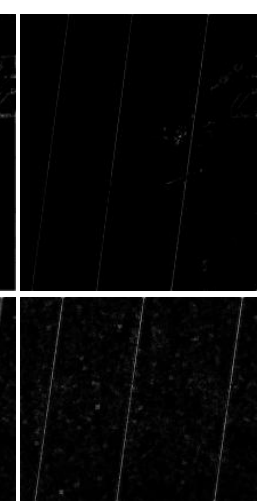

(c)
Figure 2. Examples of ridge point detection

\subsection{Line Feature Extraction}

At this stage the ridge points have been detected as candidate power lines, but the number of power lines and which ridge points belong to power lines are still unknown. Not all linear features are power lines, so these noise linear features should be removed as they will affect the computation of the direction and position of the power lines. The pose of the flying UAV is adjusted based on power lines captured by the on-board Gimbal camera, so the direction and position of the power lines are critical to guide UAV flying directly above the power lines. In this section, we will focus on how to extract power lines from the candidate ridge points.

Hough transform (HT) is the most widely used method for line detection. The major drawback of the HT is the multiple response of a line and detection of infinite lines on edge map with one pixel width, which makes it unsuitable for power line 
detection (Fernandes \& Oliveira, 2008). Inspired by (Von Gioi, et al., 2010), the idea of region growing and connected component analysis are adopted in this paper. The connected ridge points are grouped into a connected region, and treated as a subset of power lines. As the power line region is homogeneous, if the difference between the pixel value and the average pixel value is smaller than a threshold, that pixel belongs to the same power line region. The merging process is repeated until no new pixel can be added.

Each connected region is a line, and its orientation and position can be calculated from its covariance matrix and the eigenvalues (Lee, et al., 2006). Let $R_{i}=\left\{p_{j}\left(x_{j}, y_{j}\right) \mid j=\right.$ $1,2, \ldots, m$ be the $i^{\text {th }}$ connected region with $m$ pixels, its covariance matrix is computed by

$$
\begin{aligned}
& \mathrm{C}=\left(\begin{array}{ll}
\mathrm{c}_{11} & \mathrm{c}_{12} \\
\mathrm{c}_{21} & \mathrm{c}_{22}
\end{array}\right) \quad \mathrm{c}_{11}=\frac{1}{\mathrm{~m}} \sum_{\mathrm{j}=1, \ldots, \mathrm{m}}\left(\mathrm{x}_{\mathrm{j}}-\mathrm{x}_{\mathrm{m}}\right)^{2} \\
& \mathrm{c}_{12}=\mathrm{c}_{21}=\frac{1}{\mathrm{~m}} \sum_{\mathrm{j}=1, \ldots, \mathrm{m}}\left(\mathrm{x}_{\mathrm{j}}-\mathrm{x}_{\mathrm{m}}\right)\left(\mathrm{y}_{\mathrm{j}}-\mathrm{y}_{\mathrm{m}}\right) \\
& \mathrm{c}_{22}=\frac{1}{\mathrm{~m}} \sum_{\mathrm{j}=1, \ldots, \mathrm{m}}\left(\mathrm{y}_{\mathrm{j}}-\mathrm{y}_{\mathrm{m}}\right)^{2}
\end{aligned}
$$

where $x_{m}, y_{m}=$ the centroid of the connected region $R_{i}$,

$$
\mathrm{x}_{\mathrm{m}}=\frac{1}{\mathrm{~m}} \sum_{\mathrm{j}=1, \ldots, \mathrm{m}} \mathrm{x}_{\mathrm{j}}, \mathrm{y}_{\mathrm{m}}=\frac{1}{\mathrm{~m}} \sum_{\mathrm{j}=1, \ldots, \mathrm{m}} \mathrm{y}_{\mathrm{j}}
$$

The line angle $\theta$ is defined as

$$
\theta=\tan ^{-1} \frac{\left(\lambda_{1}-c_{11}\right)}{c_{12}}=\tan ^{-1} \frac{c_{21}}{\left(\lambda_{1}-c_{22}\right)}
$$

where $\quad \lambda_{1}=$ small eigenvalue of the covariance matrix

After detection of connected components, collinear line segments are linked to form a long line by using the line fitting algorithm. Figure 3 shows an example of line detection result.

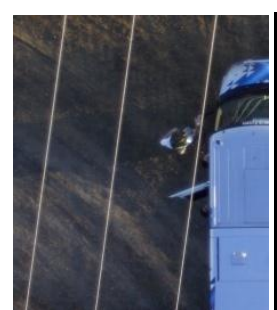

(a)

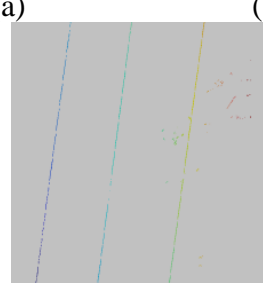

(d)

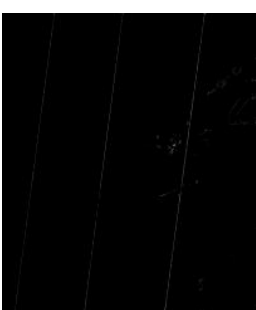

(b)

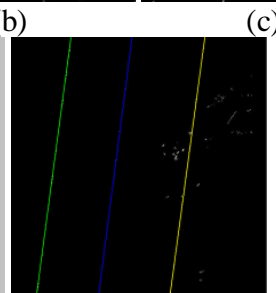

(e)

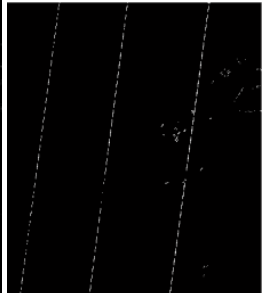

(c)
Figure 3. Linear feature detection result. (a) original image; (b) ridge points; (c)-(d) connected components and their colour representation; (e) detected power lines

\section{ACTIVE UAV GUIDANCE}

The active guidance proposed in this paper assumes that the aircraft has a Gimbal camera with at least two degrees of freedom (e.g pan and tilt). The aircraft is guided through the implementation of two control loops, Gimbal and aircraft respectively. We first control the position of the Gimbal such that the power line is in the field of view of the camera $(\theta=0$ and $\left.T_{1}=0\right)$. To accomplish this control task we extract two variables from the lines. Their distance to the image centre $\left(T_{1}\right)$ and their orientation with regards to the vertical $(\theta)$. Figure 4 illustrates the estimated variables in the image plane.

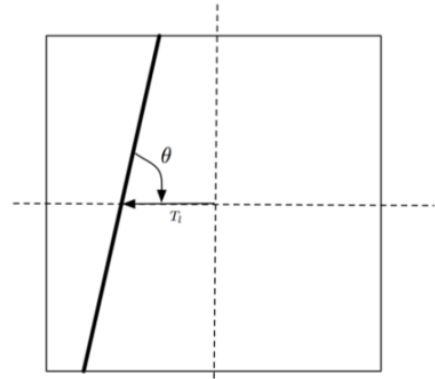

Figure 4. Variables obtained from the image plane for control perpose. $\theta$ is the angle with regards to the vertical and $T_{1}$ is the distance to the image center

Once the Gimbal is actively tracking the power line. Its pose is then used to generate the appropriate control commands such that the aircraft moves and flies above the lines. This is achieved comparing the actual Gimbal pose with the desired pose (90 deg tilt and 0 deg pan). The desired Gimbal pose is seen as the pose the Gimbal would have if the aircraft was at flight level flying directly above the power lines with the Gimbal looking downward. A high level control architecture is shown in Figure 5 in which dotted-lines represents the crosscoupling between the aircraft orientation, the Gimbal pose and the position of the features in the image planes.

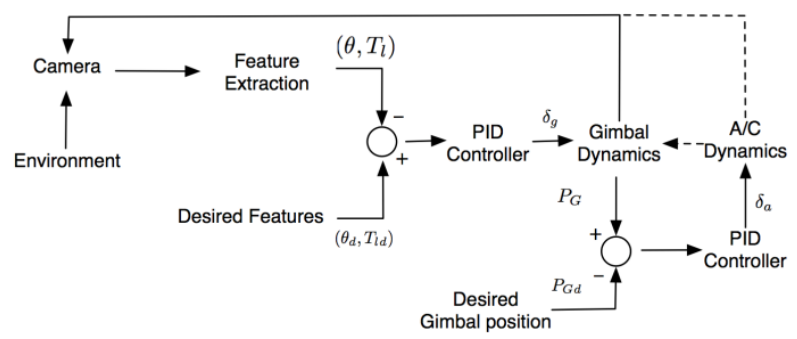

Figure 5. High level control architecture for the active guidance. Sub-index $\boldsymbol{d}$ denotes desired values and $\boldsymbol{\delta}$ are the commanded values.

Overall the control task will command the Gimbal and aircraft simultaneously to achieve the desired flight trajectory (directly above the power lines).

\section{EXPERIMENTS AND RESULTS}

This section presents the experimental results. A number of aerial images taken from our flight trials with an UAV have been used in the test. The images were taken at altitude ranging from 20 to 60 meters above power lines. A power line in an image is represented as at least 2 pixels.

Two state-of-the-art line detection methods, LSD (Von Gioi, et al., 2010) and EDLines (Akinlar \& Topal, 2011), are used to as baseline to evaluate the proposed algorithm. Both LSD and EDLines detect lines based on edges. To illustrate the advantage of using ridges in line detection, the results on a 
synthetic image is show in Figure 6. From left to right the input image with a thick line, line detection with our algorithm, and line detection with EDLine are shown as (a), (b) and (c) respectively. From the result, it is obvious that for a line represented as more than 2 pixels, edge based line detection produces two edges lines. While for ridge based line detection there is only one response for a line.

Figure 7 shows more comparison results of our algorithm and the two baseline algorithms in real images. Each row in Figure 7 represents a test set and the columns from left to right are the input image and the results of EDLines, LSD and our algorithm respectively. Overall, the proposed algorithm obtained better detection results with less false positive lines than the EDLines and LSD. Moreover, as stated before, EDLines and LSD cannot be used to estimate the number of power line as they are based on edges. However, it is noted that some fence lines (Figure 7 (d)) and road centrelines (Figure 7 (c)) have also been detected. Discriminating these very similar line features from power lines could be very difficult given no elevation clues can be used from image only. A line feature tracking scheme in the power line detection process will be helpful in the future.

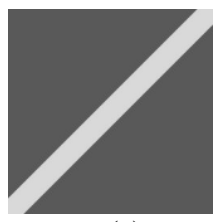

(a)

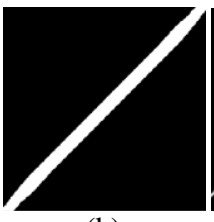

(b)

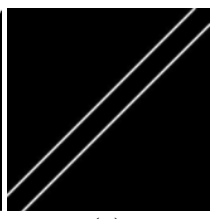

(c)
Figure 6. Illustration of ridge-based and edge-based line detection on a synthetic image

To achieve real-time vision-based UAV guidance, the processing speed of the computer vision algorithm is of great importance. Compared to EDLines and LSD, the proposed algorithm is much faster because the line fitting are conducted in the filtered image using steerable filters. While line fitting and pixel searching are applied to the all edges for EDLines and time-consuming region growing method is applied on the gradient map of the whole image for LSD.

Table 1. Comparison of computational time

\begin{tabular}{c|c|c|c|c}
\hline Image & Size & EDLines (ms) & LSD (ms) & $\begin{array}{c}\text { Our algorithm } \\
(\mathrm{ms})\end{array}$ \\
\hline (a) & $477 \times 411$ & 12.4 & 100 & 3.4 \\
(b) & $604 \times 704$ & 12.4 & 100 & 3.9 \\
(c) & $566 \times 369$ & 9.2 & 800 & 2.1 \\
(d) & $960 \times 960$ & 40.6 & 1400 & 14.7 \\
(e) & $1178 \times 714$ & 44.4 & 1600 & 19.2 \\
(f) & $1111 \times 685$ & 37.7 & 1200 & 17.3 \\
\hline
\end{tabular}

\section{CONCLUSION}

This paper presented a fast power line detection method and a UAV active guidance control concept. The two steps involved in the proposed detection algorithm are: 1) identifying ridge points from the energy functions of a steerable filter; 2) line fitting on the detected ridge points using connected component analysis. The proposed algorithm can reduce false positive lines and is computationally efficient. The experimental results against two state-of-the-art line detection algorithms demonstrate the performance of the proposed algorithm.

\section{ACKNOWLEDGEMENTS}

This research has been supported by the Cooperative Research Centre for Spatial Information, whose activities are funded by the Australian Government's Cooperative Research Centres Programme. This work is the result of a collaboration between the Australian Research Centre for Aerospace Automation (ARCAA) at Queensland University of Technology (QUT) and Ergon Energy Australia

\section{REFERENCES}

Akinlar, C. \& Topal, C., 2011. Edlines: A real-time line segment detector with a false detection control. Pattern Recognition Letters, 32(13), pp. 1633-1642.

Cai, J., 2011. Robust thinning algorithm without artefacts for pattern recognition and $3 \mathrm{~d}$ plant modelling. In: Proceedings of 2011 International Conference on Multimedia Technology (ICMT), pp. 702-706.

Casasent, D.P. \& Smokelin, J.-S., 1994. Real, imagnary and clutter gabor filter fusion for detection with reduced false alarms. Optical engineering, 33(7), pp. 2255-2263.

Fernandes, L.A.F. \& Oliveira, M.M., 2008. Real-time line detection through an improved hough transform voting scheme. Pattern Recognition, 41, pp. 299-314.

Hough, P.V.C., 1962. A method and means for recognizing complex patterns. US Patent: 3,069,654.

Jang, J.-H. \& Hong, K.-S., 2002. Detection of curvilinear structures and reconstruction of their regions in gray-scale images. Pattern Recognition, 35, pp. 807-824.

Koller, T.M., et al., 1995. Multi-scale detection of curvilinear structures in 2-d and 3-d image data. In, pp. 864-869.

Lee, Y.-S., et al., 2006. A straight line detection using principal component analysis. Pattern Recognition, 27, pp. 1744-1754.

Li, Z., et al., 2010. Advances in vegetation management for power line corridor monitoring using aerial remote sensing techniques. In: Proceedings of the First International Conference on Applied Robotics for the Power Industry (CARPI), Montreal, Canada.

Liu, L. \& Dai, T.-S., 2006. Ridge orientation estimation and verification algorithm for fingerprint enhancement. Journal of Universal Computer Science, 12(10), pp. 1426-1438.

Mehrotra, R., et al., 1992. Gabor filter-based edge detection. Pattern Recognition, 25(12), pp. 1479-1493.

Mills, S., et al., 2010. Vision based control for fixed wing uavs inspecting locally linear infrastructure using skid-to-turn maneuvers. Journal of Intelligent and Robotic Systems, 61(1-4), pp. 29-42.

Nieto, M., et al., 2011. Line segment detection using weighted mean shift procedureson a $2 \mathrm{~d}$ slice sampling strategy. Pattern Anal Applic, 14, pp. 149-163. 
International Archives of the Photogrammetry, Remote Sensing and Spatial Information Sciences, Volume XXXIX-B3, 2012 XXII ISPRS Congress, 25 August - 01 September 2012, Melbourne, Australia

Rathinam, S., et al., 2008. Vision-based monitoring of locally linear structures using an unmanned aerial vehicle. Journal of Infrastructure Systems, 14(1), pp. 52-63.

Steger, G., 1998. An unbiased detector of curvilinear structures. IEEE Transactions on Pattern Analysis and Machine Intelligence, 20(2), pp. 113-125.

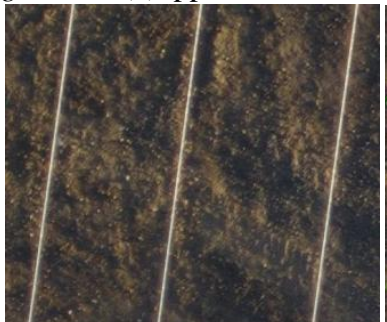

$\mathrm{a}(1)$

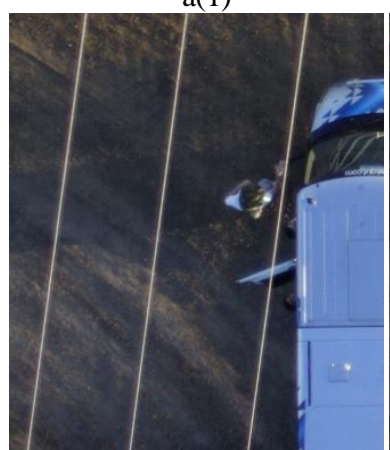

$b(1)$

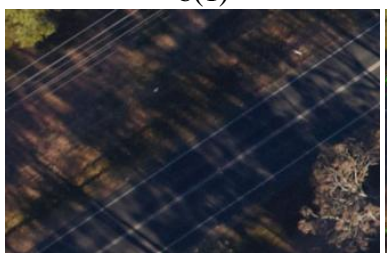

$\mathrm{c}(1)$

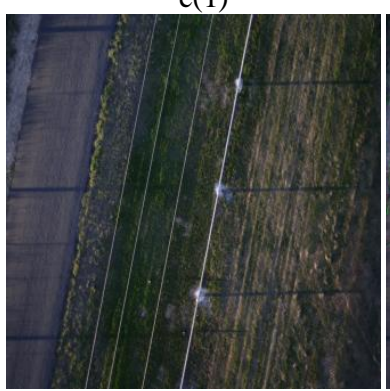

$\mathrm{d}(1)$

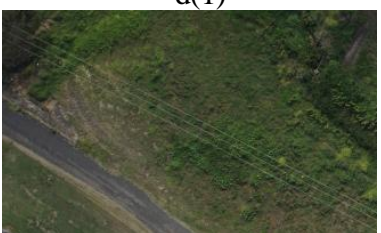

$\mathrm{e}(1)$

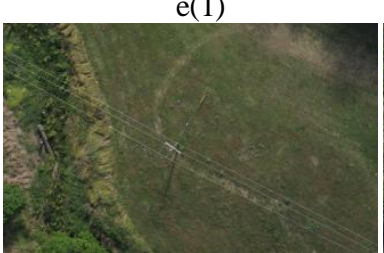

$\mathrm{f}(1)$

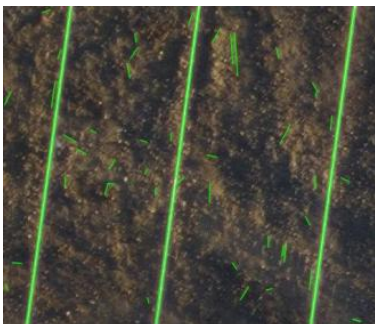

$\mathrm{a}(2)$

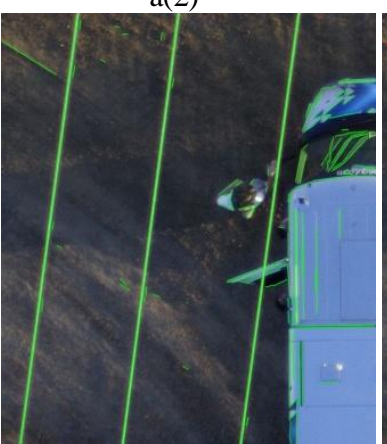

$b(2)$

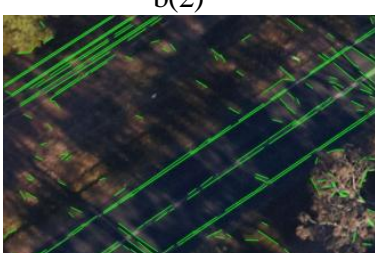

$c(2)$

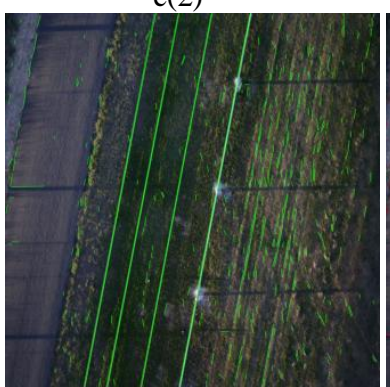

$\mathrm{d}(2)$

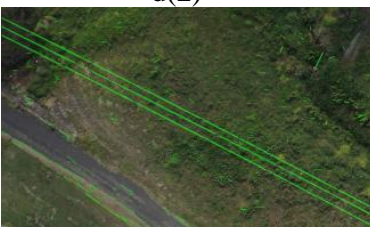

$\mathrm{e}(2)$

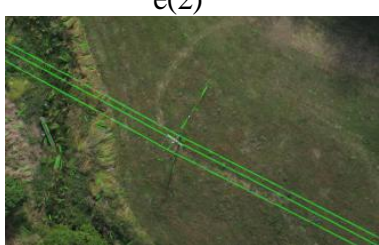

$\mathrm{f}(2)$
Von Gioi, R.G., et al., 2010. Lsd: A fast line segment detector with a false detection control. IEEE Transactions on Pattern Analysis and Machine Intelligence, 32(4), pp. 722-732.

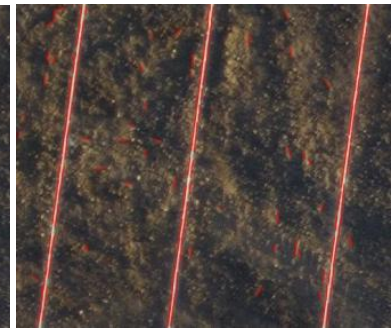

$\mathrm{a}(3)$

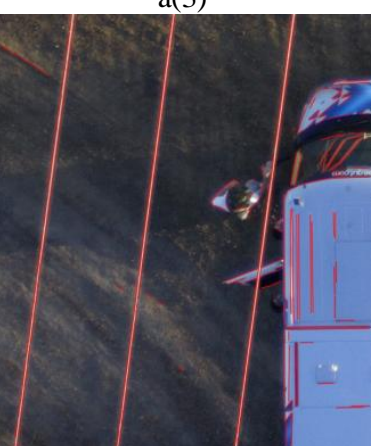

$b(3)$

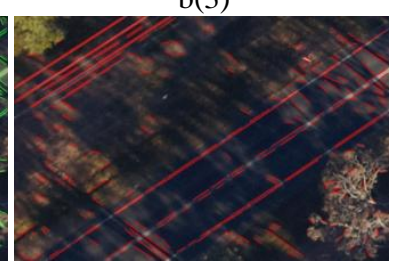

$c(3)$

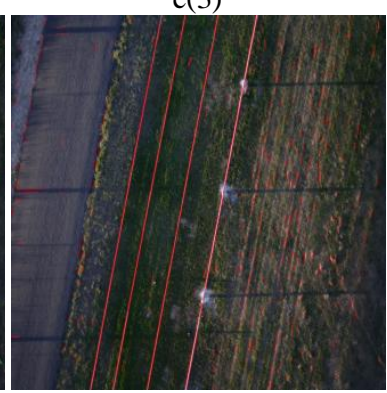

$\mathrm{d}(3)$

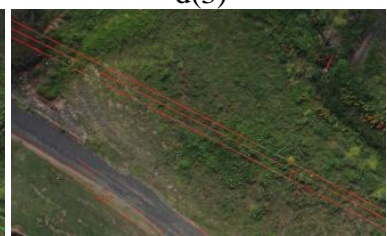

$\mathrm{e}(3)$

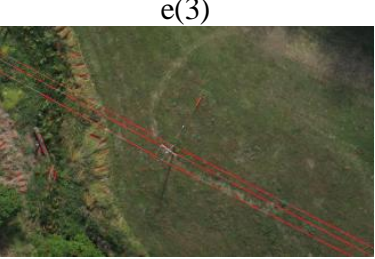

$\mathrm{f}(3)$

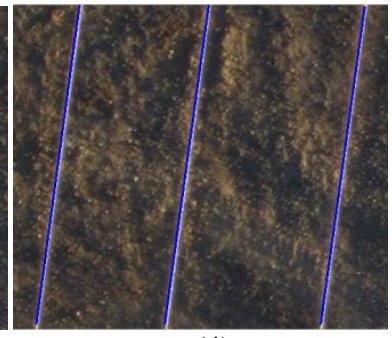

$\mathrm{a}(4)$

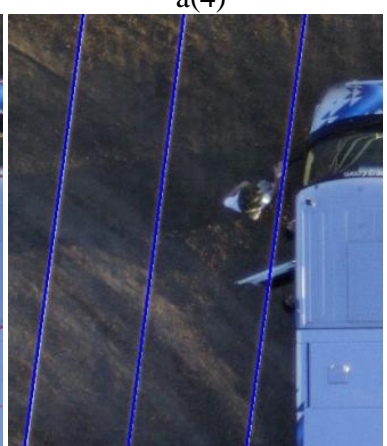

$\mathrm{b}(4)$

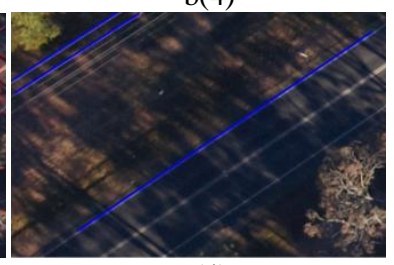

$c(4)$

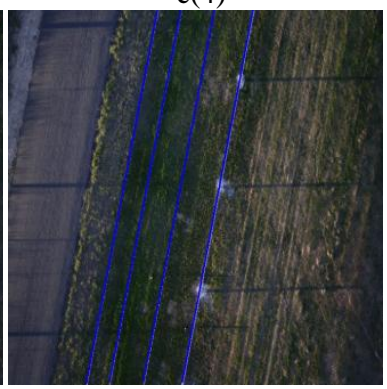

$\mathrm{d}(4)$

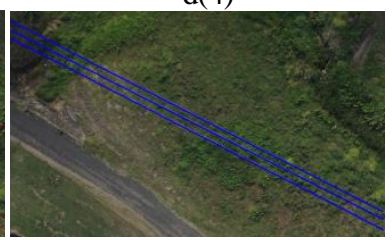

e(4)

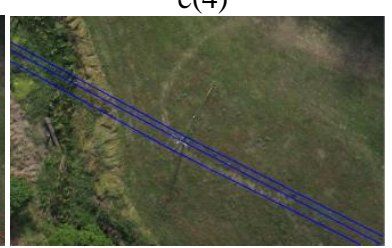

f(4)

Figure 7. Results Comparison: each row represents a test set, column 1 are the original images, column 2-4 are the detection results by EDLines, LSD and our algorithm respectively. 